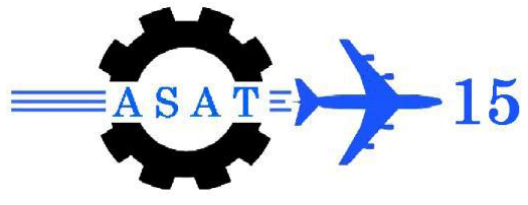

\title{
Modeling and Forced Vibration of Smart Piezoelectric Beams Using Simple Higher Order Shear Deformation Theory
}

\author{
M. A. Elshafei ${ }^{\star}$, F. Alraiess ${ }^{\dagger}$
}

\begin{abstract}
In the current work, a finite element formulation is developed for modeling and forced vibration of isotropic as well as orthotropic composite beams with distributed piezoelectric actuators subjected to both mechanical and electrical loads. The proposed model is developed based on a simple higher order shear deformation theory where the displacements field equations in the model accounts for a parabolic distribution of the shear strain and the non-linearity of in-plane displacements across the thickness and subsequences the shear correction factor is not involved. The virtual displacement method is used to formulate the equations of motion of the structure system. The model is valid for both segmented and continuous piezoelectric elements which can be either surface bonded or embedded in the laminated beams. A two-node element with four mechanical degrees of freedom in addition to one electrical degree of freedom for each node is used in the finite element formulation. The electric potential is considered as function of the thickness and the length of the beam element. The steady state responses for damped and undamprd beams are formulated. A MATLAB code is developed to compute the static deformation, free vibration parameters, and the beams responses due to harmonic excitation of mechanical and electrical loads. The predictions from the proposed model are in good shape.
\end{abstract}

Keywords: Finite element, Piezoelectric materials, higher order deformation theory, composite material mechanics, forced vibration.

\section{Nomenclature}

A

$A_{i j}$

$B_{i j}$

$b$

$C_{i j k l}$

$c_{1}, c_{2}, c_{3}$ and $c_{4}$

$\{D\}$

$\{E\}$

$E_{1}$, and $E_{2}$

$[e]$
Beam cross section area.

Elements of extensional stiffness matrix.

Elements of coupling stiffness matrix.

Width of beam element.

Elastic constants.

Constant coefficients.

Electric displacement vector $\left(\mathrm{C}_{\mathrm{m}} \mathrm{m}^{2}\right)$.

Electric field vector $(\mathrm{V} / \mathrm{m})$.

Young's modulus in the axial and transversal directions.

Piezoelectric constants tensor $\left(\mathrm{C} / \mathrm{m}^{2}\right)$.

\footnotetext{
*Egyptian Armed Forces, Egypt, melshafei@yahoo.com

${ }^{\dagger}$ Libyan Armed Forces, Libya.
} 


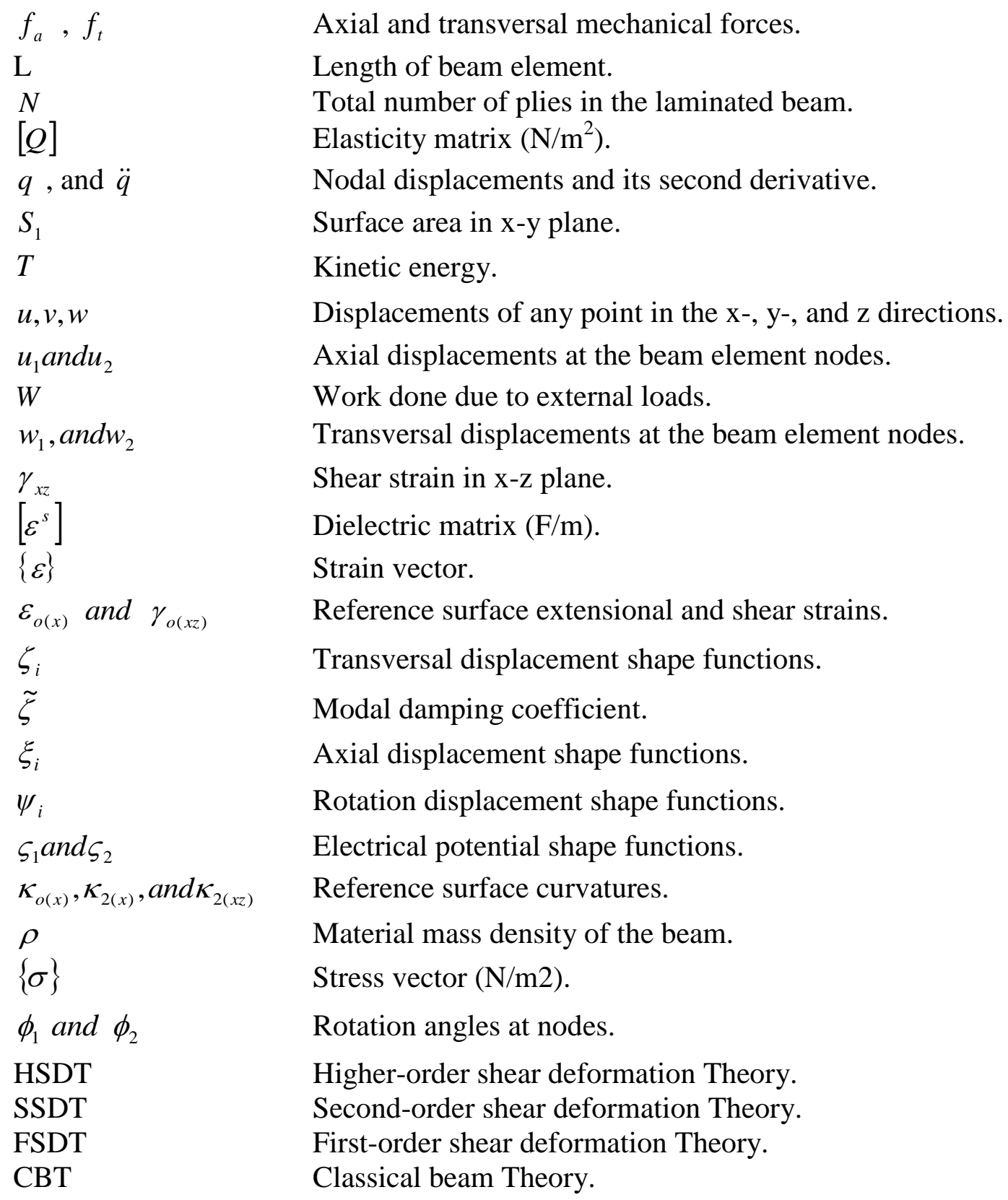

\section{Introduction}

Several researchers have studied the forced vibration and dynamic analysis of smart structures with piezoelectric material.

KYU Kang al., 1996, proposed finite element model to predicte theoretically and measured experimentally the damping factor and model damping of the carbon/epoxy beams with piezoelectric materials. They suggested that the modal damping was more appropriate performance index rather than the damping ratio for vibration suppression in the structure from fasting point of view. Samanta et al., 1996, proposed a finite element formulation based on (HODT) for active vibration control of composite plate with piezoelectric actuators and sensors due to the applied harmonic mechanical and electrical loads. They concluded that significant reduction in vibration amplitude occurs because of increase of induced damping through feedback. Wang and Quek, 2000, provided model for flexural and free vibration analysis of sandwich beam with piezoelectric layer. They concluded that the dynamic characteristics of the entire structure are related to the position of the piezoelectric layer. Sun and Huang, 2000, derived analytical solution of smart composite beams based on (FODT) 
(Mindlin plate theory). They found that the displacement decay amplitude increases as the feedback control gain increases, and the piezoelectric film (PVDF) has a 'breakdown' voltage of around $200 \mathrm{~V}$. When the input control voltage exceeds this breakdown voltage, the dipolar molecular structure of the PVDF is destroyed.

Hwu et al., 2001, derived an orthogonality relation contained the effects of rotary inertia and shear deformation for the free and forced vibrations of smart composite sandwich beams. The effects of these two factors are illustrated by numerical examples to complete their study. Mitra et al., 2004, presented finite element model based on (FODT) of composite thin walled box beams with piezoelectric actuators for vibration control. Open and closed loop responses were obtained using state space modeling. A proportional-integral (PI) controller using acceleration feedback was implemented for control the vibration due to single-frequency excitations. Their experimental and numerical results correlated very well in the proposed analysis. Senthil, and Baillargeon, 2004, proposed finite element model of sandwich cantilever beam and laminated composite plates using piezoelectric actuators/sensors for active vibration suppression. The electric field was applied perpendicular to the direction of polarization. Their predictions showed good agreement with the experimental results. ZHOU et al., 2005, developed analytical model for piezoelectric bimorph based on improved (FSDT). Their model combined the equivalent single-layer approach for mechanical displacements and a layer wise-type modeling of the electric potential. The obtained results of their model revealed that: i) Excellent agreement between the model predictions with shear correction factor equal to 8/9 and the exact solutions for the resonant frequencies. ii) Accurate bending vibration frequencies even for thick beam, whereas the classical thin beam theory or plate theory gives low accurate results. iii) Further investigations are needed for the shear correction factor of the piezoelectric laminates. Trindade and Maio, 2006, developed finite element formulation to evaluate the vibration damping performance of sandwich cantilever beams. The beams connected to resistive shunt circuits for the passive vibration control. The obtained results for cantilever beam gave a reduction up to $15 \mathrm{~dB}$ in resonant vibration amplitude of the third mode.

In the present work, a finite element model is developed for isotropic and orthotropic beams with piezoelectric actuators based on a simple higher order shear deformation theory made by (Reddy, 1984). The steady state response of the beam system is analyzed for six cases of loading conditions. The model is able to compute static deformations, natural frequencies, mode shapes, and the beams responses due to a harmonic excitation of both mechanical and electrical loads.

\section{Theoretical Formulation}

The displacements field equations for the beam using higher order shear deformation theory (Reddy, 1984; Khdeir and Reddy, 1997) at any point through the thickness are presented by:

$$
\begin{gathered}
u(x, y, z)=u_{\circ}(x)+z \phi_{x}-\frac{4}{3 h^{2}} z^{3}\left[\phi_{x}+\frac{\partial w}{\partial x}\right] \\
v(x, y, z)=0 \\
w(x, y, z)=w_{\circ}(x)
\end{gathered}
$$

where $u_{0}$ and $w_{0}$ denote the displacements of a point $(x, y, 0)$ at the mid plane, and $\phi(x)$ is the rotation angle along $x$ axis. For one-dimensional beam where the width in the $y$ direction is stress free and by using the plane stress assumption the remaining strains components $\varepsilon_{x x}$ and $\gamma_{x z}$ are represented by: 


$$
\begin{gathered}
\varepsilon_{x x}(x, y, z) \equiv \frac{\partial u(x, z)}{\partial x}=\frac{\partial u}{\partial x}+z \frac{\partial \phi_{x}}{\partial x}-z^{3} \frac{4}{3 h^{2}}\left[\frac{\partial \phi_{x}}{\partial x}+\frac{\partial^{2} w}{\partial x^{2}}\right]=\varepsilon_{o(x)}+z \kappa_{\mathrm{o}(x)}+z^{3} \kappa_{2(x)} \\
\gamma_{x z}(x, y, z) \equiv \frac{\partial w(x, y, z)}{\partial x}+\frac{\partial u(x, y, z)}{\partial z}=\frac{\partial w}{\partial x}+\phi_{x}-z^{2} \frac{4}{h^{2}}\left[\phi_{x}+\frac{\partial w}{\partial x}\right]=\gamma_{o(x z)}+z^{2} \kappa_{2(x z)}
\end{gathered}
$$

\section{Piezoelectric Constitutive Relations}

The piezoelectric constitutive equations are given by (Nye, 1985; Tzou and Tseng, 1990; Ikeda, 1996):

$$
\begin{gathered}
\sigma_{i j}=c_{i j k l} \varepsilon_{k l}-e_{k i j} E_{k} \\
D_{i}=e_{i k l} \varepsilon_{k l}+\varepsilon_{i k}^{s} E_{k}
\end{gathered}
$$

where; $i, j=1, . ., 6$; and $k=1, . ., 3$.

In the proposed model the following assumptions are used: (1) The width in y direction is stress free and the plane stress assumption is used. Therefore, it is possible to set $\sigma_{22}=\sigma_{23}=\sigma_{12}=\gamma_{23}=\gamma_{12}=0$ and $\varepsilon_{22} \neq 0$ in Eqn. (4). (2) The polarization axis z is aligned with the thickness direction of the beam, thus only $D_{z}$ in Eqn. (5) is taken into consideration. (3) By introducing $E_{z}$ applied across the actuator thickness and the other components of the electric fields are zeros. (4) The coefficient $e_{15}$ and $\varepsilon_{11}^{s}$ are neglected. Therefore the constitutive relations Eqn. (4) and Eqn. (5) are reduced to:

$$
\left\{\begin{array}{l}
\sigma_{x x} \\
\sigma_{x z} \\
D_{z}
\end{array}\right\}_{k}=\left[\begin{array}{ccc}
\tilde{Q}_{11} & 0 & 0 \\
0 & \tilde{Q}_{55} & 0 \\
\tilde{e}_{31} & 0 & 0
\end{array}\right]_{k}\left\{\begin{array}{c}
\varepsilon_{x x} \\
\gamma_{x z} \\
0
\end{array}\right\}_{k}-\left[\begin{array}{c}
\tilde{e}_{31} \\
0 \\
-\tilde{\varepsilon}_{z z}^{s}
\end{array}\right] E_{z}
$$

where, the coefficients in Eqn. (6) are given by:

\section{Case I: Isotropic Beam}

$$
\tilde{Q}_{11}=E \quad \tilde{Q}_{55}=G \quad \text {, and } \quad \bar{Q}_{i j}=Q_{i j}
$$

\section{Case II: Orthotropic Beam}

$$
\tilde{Q}_{11}=\bar{Q}_{11}-\frac{\bar{Q}_{12} \bar{Q}_{12}}{\bar{Q}_{22}} \quad \tilde{Q}_{55}=\bar{Q}_{55}-\frac{\bar{e}_{25}}{\bar{e}_{24}} \bar{Q}_{45}
$$

And the piezoelectric coefficients are given by:

$$
\tilde{\varepsilon}_{z z}^{s}=\bar{\varepsilon}_{z z}^{s}+\frac{\bar{e}_{32} \bar{e}_{32}}{\bar{Q}_{22}} \quad \tilde{e}_{31}=\bar{e}_{31}-\bar{e}_{32} \frac{\bar{Q}_{12}}{\bar{Q}_{22}} \quad \tilde{e}_{15}=\bar{e}_{15}-\frac{\bar{e}_{25} \bar{e}_{14}}{\bar{e}_{24}}
$$

where; $\bar{Q}_{i j}$ and $\bar{e}_{i j}$ are the transformed reduced stiffness coefficients, and piezoelectric modules, respectively (Reddy, 2004). 


\section{Energy Formulation}

The kinetic energy of the structure system is given by:

$$
T=\frac{1}{2} \int_{v} \rho\left[\dot{u}^{2}+\dot{w}^{2}\right] d v
$$

The work done due to external mechanical and electrical loads is represented by:

$$
W=\int_{0}^{L} f_{a} u d x+\int_{0}^{L} f_{t} w d x+f_{t_{i}} w_{i}-\int_{S_{1}} \sigma \varphi d S_{1}
$$

where; $\sigma\left(\mathrm{C} / \mathrm{m}^{2}\right)$ is the surface charge density on the actuator surface, and $\varphi$ is the electric potential at the piezoelectric surface area $S_{1}$ at $\left(z_{1}=h_{1}\right)$.

The total internal strain energy for the structure system $U$ is the sum of internal strain energy, and the electric field potential energy such as (Tzou and Tseng, 1990):

$$
U=\frac{1}{2} \int_{v}\left[\left(\sigma_{x x} \varepsilon_{x x}+\sigma_{x z} \gamma_{x z}\right)-\left(D_{z} E_{z}\right)\right] d v
$$

\section{Case I: Isotropic Beam}

$$
U=\frac{1}{2} \int_{v}\left[E \varepsilon_{x x}^{2}+G \gamma_{x z}^{2}-2 \tilde{e}_{31} E_{z} \varepsilon_{x x}-\widetilde{\varepsilon}_{z z}^{s} E_{z}^{2}\right] d v
$$

\section{Case II: Orthotropic Beam}

$$
U=\frac{1}{2} \int_{v}\left[\left(\tilde{Q}_{11} \varepsilon_{x x}^{2}+\tilde{Q}_{55} \gamma_{x z}^{2}-2 \tilde{e}_{31} E_{z} \varepsilon_{x x}-\tilde{\varepsilon}_{z z}^{s} E_{z}^{2}\right)\right] d v
$$

\section{Finite Element Formulation}

A two nodes beam element with four mechanical degrees of freedom and one electrical degree of freedom $\{q \phi\}^{T}=\left\{u, w, \frac{\partial w}{\partial x}, \varphi_{x}, \phi\right\}^{T}$ at each node are used.

$$
\left\{u, w, \frac{\partial w}{\partial x}, \phi_{x}, \varphi\right\} \quad\left\{u, w, \frac{\partial w}{\partial x}, \phi_{x}, \varphi\right\}
$$

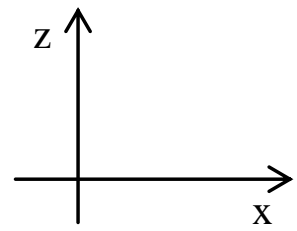

\section{Figure 1. Element nodal degrees of freedoms.}

The linear interpolation shape functions $\xi_{j}$ and $\psi_{j}$ are used for representing the axial displacement $u_{0}$ and the rotation angle $\phi_{x}$ at the mid-plane as (Cook et al., 1974):

$$
\begin{aligned}
& \xi_{1}=1-\frac{x}{L}, \text { and } \xi_{2}=\frac{x}{L} \\
& \psi_{1}=1-\frac{x}{L}, \text { and } \psi_{2}=\frac{x}{L}
\end{aligned}
$$

And a Hermit cubic shape functions $\zeta_{j}$ are used for representing the transversal displacement $w_{0}$ at the mid-plane as (Cook et al., 1974): 


$$
\begin{array}{cc}
\zeta_{1}=1-3\left(\frac{x}{L}\right)^{2}+2\left(\frac{x}{L}\right)^{3} & \zeta_{2}=x-2\left(\frac{x^{2}}{L}\right)+\frac{x^{3}}{L^{2}} \\
\zeta_{3}=3\left(\frac{x}{L}\right)^{2}-2\left(\frac{x}{L}\right)^{3} & \zeta_{4}=-\frac{x^{2}}{L}+\frac{x^{3}}{L^{2}}
\end{array}
$$

In the proposed model the electric potential is considered as function of the thickness and the length of the beam (Elshafei ${ }^{1}$ et al., 2010; Elshafei ${ }^{2}$ et al., 2013). In case of the electric potential is function of the length, it can be represented by:

$$
\varphi(x)=\varphi_{1} \widehat{\varsigma}_{1}+\varphi_{2} \widehat{\varsigma}_{2}=\sum_{j=1}^{2} \varphi_{j} \widehat{\varsigma}_{j}
$$

where;

$$
\widehat{\varsigma}_{1}=1-\frac{x}{L}, \text { and } \quad \widehat{\varsigma}_{2}=\frac{x}{L}
$$

And in case the electric potential is function of the thickness of the beam, it is given as:

where; $\quad \breve{\varsigma}_{1}=\frac{1}{2}+\frac{z}{h}, \quad \breve{\varsigma}_{2}=\frac{1}{2}-\frac{z}{h}$

Thus by the product of equations (20) and (22) and impose the homogenous boundary condition on the bottom surface to eliminate the rigid body modes. Thus the electric potential can be written as (Elshafei ${ }^{1}$ et al., 2010; Elshafei $^{2}$ et al., 2013):

$$
\varphi(x, 0, z)=\varphi_{1} \varsigma_{1}+\varphi_{2} \varsigma_{2}=\sum_{j=1}^{2} \varphi_{j} \varsigma_{j}
$$

And the shape functions are finally takes the form (Elshafei ${ }^{1}$ et al., 2010):

$$
\varsigma_{1}=\left(\frac{1}{2}+\frac{z}{h}\right)\left(1-\frac{x}{L}\right) ; \varsigma_{2}=\left(\frac{1}{2}+\frac{z}{h}\right)\left(\frac{x}{L}\right)
$$

\section{Variational Formulation}

The principle of the virtual displacement is expressed as follows:

$$
\delta U=\delta W+\delta T
$$

The first variation of the kinetic energy Eqn. (10) is expressed as:

$$
\delta T=\int_{v} \rho_{0}\left[\begin{array}{l}
\delta \dot{u}_{0}^{T}\left(\dot{u}_{0}+z \dot{\varphi}_{x}-\frac{4}{3 h^{2}} z^{3} \dot{\varphi}_{x}-\frac{4}{3 h^{2}} z^{3} \frac{d \dot{w}_{0}}{d x}\right)+ \\
\delta \dot{\varphi}_{x}^{T}\left(\left(z-\frac{4}{3 h^{2}} z^{3}\right) \dot{u}_{0}+z^{2} \dot{\varphi}_{x}-\frac{8}{3 h^{2}} z^{4} \dot{\varphi}_{x}+\frac{16}{9 h^{4}} z^{6} \dot{\varphi}_{x}+\left(-\frac{4}{3 h^{2}} z^{4}+\frac{16}{9 h^{4}} z^{6}\right) \frac{d \dot{w}_{0}}{d x}\right)+d v \\
\delta\left(\frac{d \dot{w}_{0}}{d x}\right)^{T}\left(\left(-\frac{4}{3 h^{2}} z^{3}\right) \dot{u}_{0}+\left(-\frac{4}{3 h^{2}} z^{4}+\frac{16}{9 h^{4}} z^{6}\right) \dot{\varphi}_{x}+\frac{16}{9 h^{4}} z^{6} \frac{d \dot{w}_{0}}{d x}\right)+\delta \dot{w}_{0}^{T}\left(\dot{w}_{0}\right)
\end{array}\right] d
$$

By substituting the shape functions equations (16), (17), and (18) into Eqn. (26), and perform the integration the mass matrix of the beam element can be obtained.

By inserting the displacements equation (1) into Eqn. (12) and take the first variation yields;

$$
\delta W=\int_{0}^{L} f_{a} \delta\left(u_{0}+z \phi_{x}-\frac{4}{3 h^{2}} z^{3} \phi_{x}-\frac{4}{3 h^{2}} z^{3} \frac{d w_{0}}{d x}\right) d x+\int_{0}^{L} f_{t} \delta w_{o} d x+P_{i} \delta w_{o i}-\int_{S_{1}} \sigma \delta \varphi d S_{1}
$$

By substituting the shape functions equations (16), (17), (18) and (24) into Eqn. (27) and perform the integration over the length of the beam yields the element load vector. Thus, 


\section{Case I: Isotropic Beam}

By inserting Eqns. (2) into Eqn. (14) and take the first variation, the strain energy equation (14) takes the form:

$$
\delta U=\int_{v}\left[\begin{array}{l}
{\left[\begin{array}{l}
{\left[\begin{array}{l}
\left(\frac{\partial u_{0}}{\partial x} \delta \frac{\partial u_{0}}{\partial x}\right)+z\left(\frac{\partial u_{0}}{\partial x} \delta \frac{\partial \varphi_{x}}{\partial x}+\frac{\partial \varphi_{x}}{\partial x} \delta \frac{\partial u_{0}}{\partial x}\right)+z^{2}\left(\frac{\partial \varphi_{x}}{\partial x} \delta \frac{\partial \varphi_{x}}{\partial x}\right) \\
-\frac{4}{3 h^{2}} z^{3}\left(\frac{\partial u}{\partial x} \delta \frac{\partial \varphi_{x}}{\partial x}+\frac{\partial \varphi_{x}}{\partial x} \delta \frac{\partial u}{\partial x}+\frac{\partial u}{\partial x} \delta \frac{\partial^{2} w}{\partial x}+\frac{\partial^{2} w}{\partial x} \delta \frac{\partial u}{\partial x}\right) \\
-\frac{4}{3 h^{2}} z^{4}\left(2 \frac{\partial \varphi_{x}}{\partial x} \delta \frac{\partial \varphi_{x}}{\partial x}+\frac{\partial \varphi_{x}}{\partial x} \delta \frac{\partial^{2} w}{\partial x^{2}}+\frac{\partial^{2} w}{\partial x} \delta \frac{\partial \varphi_{x}}{\partial x}\right) \\
+\frac{16}{9 h^{4}} z^{6}\left(\frac{\partial \varphi_{x}}{\partial x} \delta \frac{\partial \varphi_{x}}{\partial x}+\frac{\partial \varphi_{x}}{\partial x} \delta \frac{\partial^{2} w}{\partial x^{2}}+\frac{\partial^{2} w}{\partial x^{2}} \delta \frac{\partial \varphi_{x}}{\partial x}+\frac{\partial^{2} w}{\partial x^{2}} \delta \frac{\partial^{2} w}{\partial x^{2}}\right)
\end{array}\right]} \\
+G\left[\begin{array}{l}
\left(1-\frac{8}{h^{2}} z^{2}+\frac{16}{h^{4}}\right)\left(\left(\varphi_{x} \delta \varphi_{x}\right)+\left(\varphi_{x} \delta \frac{\partial w_{0}}{\partial x}+\frac{\partial w_{0}}{\partial x} \delta \varphi_{x}\right)+\left(\frac{\partial w_{0}}{\partial x} \delta \frac{\partial w_{0}}{\partial x}\right)\right)
\end{array}\right] \\
{\left[\begin{array}{l}
\left(\frac{\partial u_{0}}{\partial x} \delta \frac{\partial \phi}{\partial z}+\frac{\partial \phi}{\partial z} \delta \frac{\partial u_{0}}{\partial x}\right)+z\left(\frac{\partial \varphi_{x}}{\partial x} \delta \frac{\partial \phi}{\partial z}+\frac{\partial \phi}{\partial z} \delta \frac{\partial \varphi_{x}}{\partial x}\right) \\
-\frac{4}{3 h^{2}} z^{3}\left(\frac{\partial \varphi_{x}}{\partial x} \delta \frac{\partial \phi}{\partial z}+\frac{\partial \phi}{\partial z} \delta \frac{\partial \varphi_{x}}{\partial x}+\frac{\partial^{2} w}{\partial x^{2}} \delta \frac{\partial \phi}{\partial z}+\frac{\partial \phi}{\partial z} \delta \frac{\partial^{2} w}{\partial x^{2}}\right)
\end{array}\right]-\tilde{\varepsilon}_{z z}^{s}\left[\left(\frac{\partial \phi}{\partial z} \delta \frac{\partial \phi}{\partial z}\right)\right.}
\end{array}\right]}
\end{array}\right]
$$

By substituting the shape functions equations (16), (17), (18) and (24) into Eqn. (28) and perform the integration over the beam volume yields the element stiffness matrix for isotropic beam.

\section{Case II: Orthotropic Beam}

The first variation of the strain energy equation (15) takes the form:

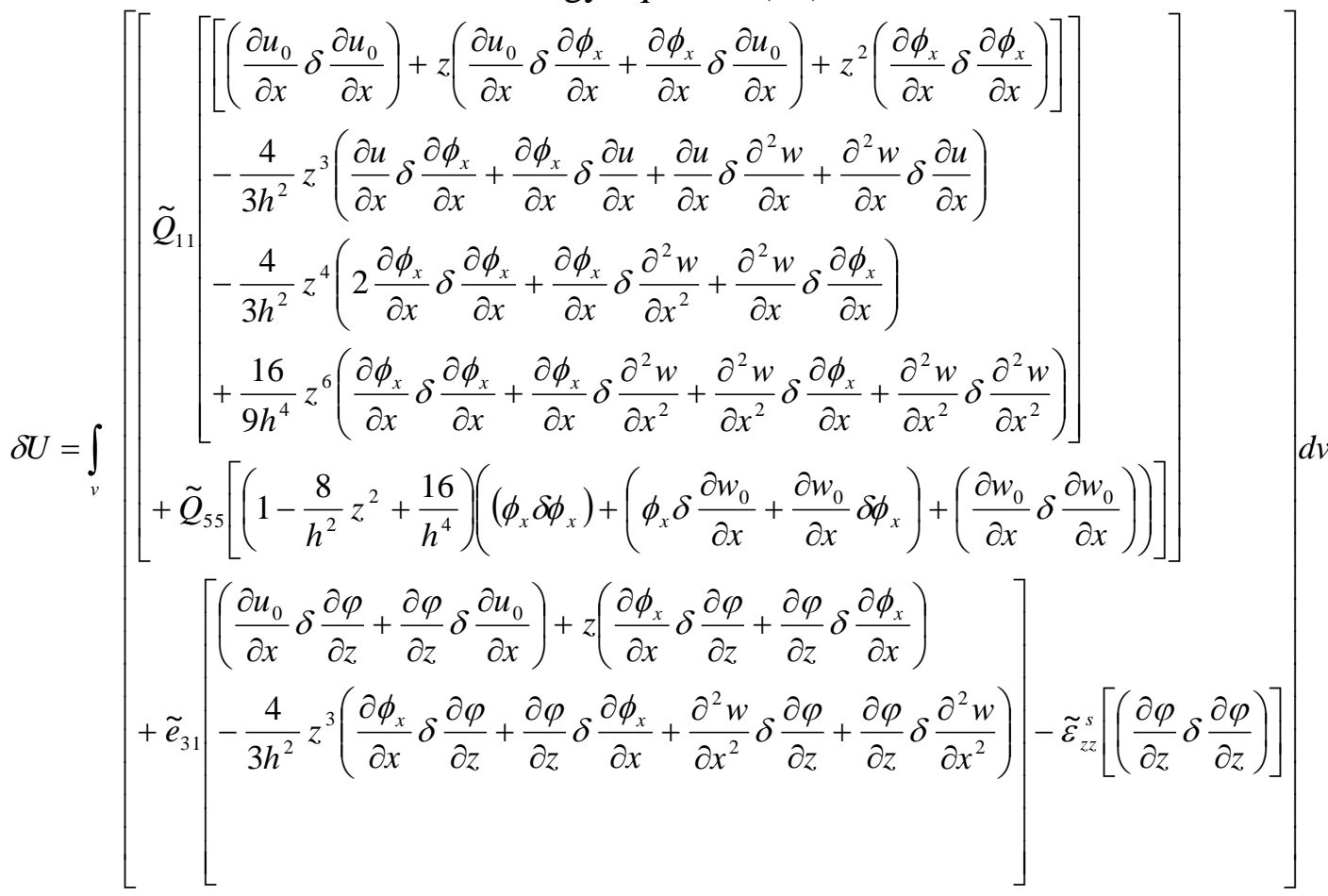


By substituting the shape functions equations (16), (17), (18) and (24) into Eqn. (29) and perform the integration over the beam with length $L$, width $b$, and height $h$ yields the element stiffness matrix for orthotropic beam.

\section{Equation of Motion}

The overall equation of motion of the structure system is obtained in terms of the global coordinates representing the global generalized mechanical displacements vector $\{q\}$, and the global generalized electric coordinate vector $\{\varphi\}$ describing the applied voltages at the actuators as follows:

$$
\left[\begin{array}{cc}
M_{q q} & 0 \\
0 & 0
\end{array}\right]\left\{\begin{array}{l}
\ddot{q} \\
\ddot{\varphi}
\end{array}\right\}+\left[\begin{array}{cc}
K_{q q} & K_{q \varphi} \\
K_{\varphi q} & K_{\varphi \varphi}
\end{array}\right]\left\{\begin{array}{l}
q \\
\varphi
\end{array}\right\}=\left\{\begin{array}{l}
F \\
G
\end{array}\right\}
$$

where $M_{q q}$ and $K_{q q}$ represent the global generalized mass and stiffness matrices corresponding to the vector of mechanical displacement. $K_{q \varphi}$, and $K_{\varphi q}$ represent the static coupling of the substrate with the piezoelectric layers. And the term $K_{\varphi \varphi}$ is electric stiffness matrix. The vector $\{F\}$ is the applied mechanical force vector, and $\{G\}$ is the electric excitation force vector as a result of the applied surface charge density distribution on the actuators.

\section{Forced Vibration}

To study the forced vibration response of the structure substrate a transverse harmonic mechanical load is applied normal on the top surface, and a harmonic voltage is also applied to the actuator layer. Both of these loads are represented by:

$$
f_{t}(z, t)=f_{0} \sin \omega t \text { And } \varphi^{1}(x, 0, z, t)=V_{0} \sin \omega t
$$

where; $V_{0}, f_{0}$, and $\omega$ are the amplitude of the electrical excitation distribution (volt), the transverse mechanical loads, and the excitation frequency, respectively.

The first variation of the external work equation (18) as a function of time has the form:

$$
\delta W=\int_{0}^{L} f_{t}(z, t) \delta w d x+f_{t_{i}}(z, t) \delta w_{i}-\int_{S_{1}} \delta \delta \varphi(x, 0, z, t) d S_{1}
$$

By substituting Eqn.(31) Eqn. into (32) and the shape functions Eqns.(18), and (24) into Eqn.(32) and perform the integration over the beam length and surface of the piezoelectric actuator yields the mechanical load vector $\{F(t)\}$ and electrical actuating load vector $\{G(t)\}$, respectively in the right hand side of Eqn. (30) as a function of time. The electrical actuating load vector $\{G(t)\}$ is expressed with specified applied voltage instead of specifying the charge distribution.

The structure damping is included in the overall structure system equation, Eqn. (30), and it can be written as:

$$
[M]\{\ddot{q}\}+\left[C_{R}\right]\{\dot{q}\}+\left\lfloor K_{q q}\right\rfloor\{q\}=\{F(t)\}+\left\lfloor K_{q \varphi}\right\rfloor\{\varphi(t)\}
$$

where; $\left[C_{R}\right]$ is the Rayleigh's damping matrix and given as follows:

$$
\left[C_{R}\right]=\alpha[M]+\beta\left\lfloor K_{q q}\right\rfloor
$$

And $\alpha$ and $\beta$ are Rayleigh's coefficients of proportionality. 
The steady state response of the beam system is analyzed using mode superposition. By using left hand side of Eqn. (30) the eigenvalue /eigenvector analysis can be performed and using for uncouple the global system of ordinary differential equations. The independent ordinary differential equations could then be solved individually and the response for any given vibration model can be obtained such as:

For un-damping case:

The solution of equation (30) can be expressed as (Rao, 1995):

$$
q_{i}(t)=q_{i}(0) \cos \omega_{i} t+\frac{\dot{q}_{i}(0)}{\omega_{i}} \sin \omega_{i} t+\frac{1}{\omega_{i}} \int_{0}^{L} Q_{i}(\tau) \sin \omega_{i}(t-\tau) d \tau \quad i=1,2, \ldots \ldots, n
$$

For damping case:

The solution of equation (30/36) can be expressed as (Rao, 1995):

$$
\begin{aligned}
q_{i}(t)=q_{i}(0) e^{-\tilde{\zeta}_{i} \omega_{i} t} \cos \omega_{d} t+\left(\dot{q}_{i}(0)\right. & \left.-\frac{\tilde{\zeta}_{i}}{\sqrt{1-\tilde{\zeta}^{2}}} q_{i}(0)\right) e^{-\tilde{\zeta}_{i} \omega_{i} t} \sin \omega_{d} t \\
& +\frac{1}{\omega_{d}} \int_{0}^{L} Q_{i}(\tau) e^{-\tilde{\zeta}_{i} \omega_{i}(t-\tau)} \sin \omega_{d}(t-\tau) d \tau
\end{aligned}
$$

where; $\omega_{d}=\omega_{i} \sqrt{1-\tilde{\zeta}_{i}^{2}}$ is the damped natural frequency and $\tilde{\zeta}_{i}$ the modal damping coefficient. The initial generalized displacements and velocities $q_{i}(0)$ and $\dot{q}_{i}(0)$ can be obtained from the initial values of the physical displacements and velocities, respectively.

\section{Validation Examples}

In the present study, a MATLAB code is constructed to perform the finite element model of isotropic and orthotropic smart beams with piezoelectric materials. The static deformations, the natural frequencies, mode shapes validation are presented by (Elshafei $\left.{ }^{2}, 2013\right)$ for the proposed model. The structures responses are calculated for the beams due to different kinds of mechanical and electrical loads. The code can be used for both thick and thin composite beams. A cantilever beam consisted of structure substrate, adhesive layer, and piezoelectric layer shown in Figure 1 is used in this validation with material properties given in Table 1.
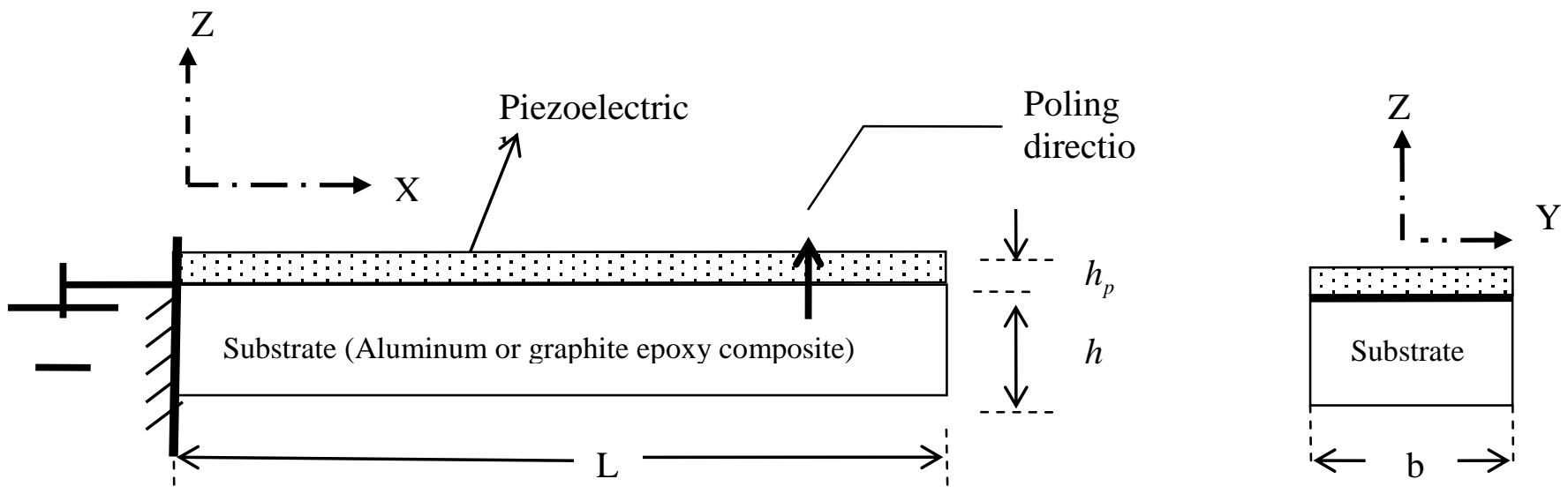
The following six cases are studied to validate the proposed forced vibration model for a beams subjected to sinusoidal electrical and mechanical loads. Both types of beams are studied in each case.

Table (1): Geometric and material properties of smart beams

\begin{tabular}{l|c|c|c|c}
\hline \hline \multicolumn{1}{|c|}{ Properties } & $\begin{array}{c}\text { Aluminum } \\
\text { substrate }^{\mathrm{a}}\end{array}$ & $\begin{array}{c}\text { A54/3501 } \\
\text { Graphite /epoxy [0] } \\
\text { composite substrate }\end{array}$ & $\begin{array}{c}\text { Adhesive } \\
\text { layer }^{\mathrm{a}}\end{array}$ & $\begin{array}{c}\text { Piezoelectric } \\
\text { actuator PZT- }^{\mathrm{a}}\end{array}$ \\
\hline$E_{11}(\mathrm{GPa})$ & 68.9 & 144.8 & 6.9 & 83 \\
\hline$E_{22}(\mathrm{GPa})$ & 68.9 & 9.65 & 6.9 & 66 \\
\hline$v_{12}$ & 0.25 & 0.3 & 0.4 & 0.31 \\
\hline$G_{12}(\mathrm{GPa})$ & 27.6 & 4.14 & 2.46 & 31 \\
\hline$d_{31}(\mathrm{~m} / \mathrm{v})$ & 0 & 0 & 0 & $-12210^{-12}$ \\
\hline$d_{33}(\mathrm{~m} / \mathrm{v})$ & 0 & 0 & 0 & $28510^{-12}$ \\
\hline$\varepsilon_{33}^{s}(\mathrm{~F} / \mathrm{m})$ & 0 & 0 & 0 & $11.5310^{-9}$ \\
\hline$\rho\left(k g / m^{3}\right)$ & 2769 & 1389.23 & 1662 & 7600 \\
\hline Length $(m)$ & 0.1524 & 0.1524 & 0.1524 & 0.1524 \\
\hline Thickness $(m)$ & 0.01524 & 0.01524 & 0.000254 & 0.001524 \\
\hline Width $(m)$ & 0.0254 & 0.0254 & 0.0254 & 0.0254 \\
\hline \hline
\end{tabular}

${ }^{\mathrm{a}}$ (Saravanos and Heyliger, 1995)

\section{A. Effect of the Mechanical and Electrical Loads Directions on the Beam Response}

Figure 3 shows the responses of aluminum beam and symmetric angle-ply laminated beam [0/90/90/0] beams due to the mechanical load with different amplitudes values $(-100 \mathrm{~N}$ and $100 \mathrm{~N}$ ) and constant applied voltage of 150 volt. It is seen from the figure that the beams responses are affected by the changing in the direction of the applied mechanical load.

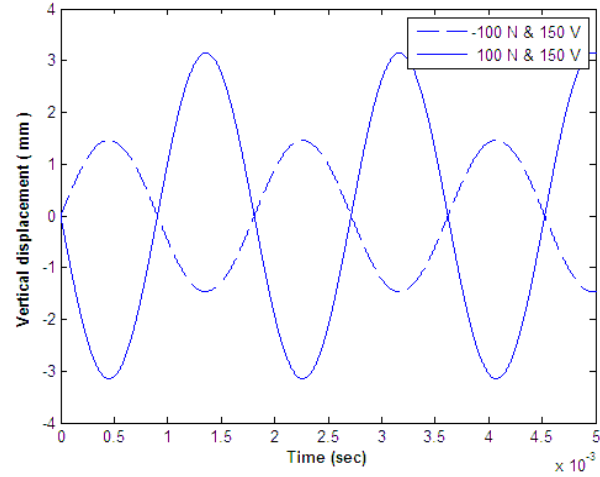

a. Aluminum beam

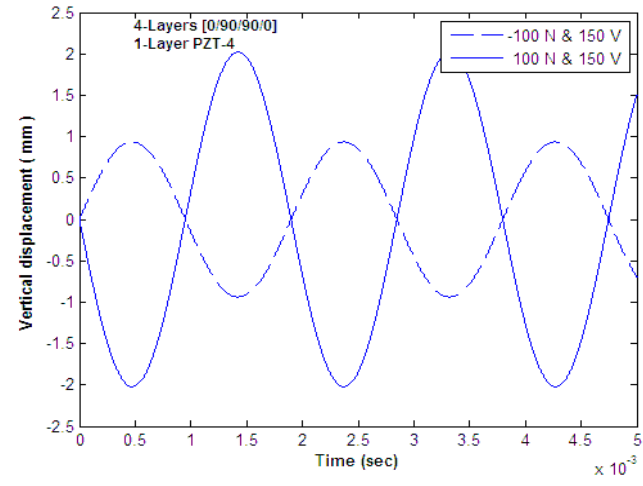

b. Composite beam

(AL Substrate -Layer PZT-4)

Figure 3: Effect of mechanical load on the beam response. 


\section{B. Effect of the Electrical Excitation Load Direction on the Beam Response at Constant Mechanical Load}

Figure 4 shows the responses of aluminum beam and symmetric angle-ply laminated beam [0/90/90/0] when the piezoelectric actuator is subjected to various values of the applied electrical loads in the polarization direction and vice versa (150 volt and -150 volt) and a constant mechanical load of $10 \mathrm{~N}$. It is seen from the figure that the beams responses are affected by the electrical excitation load direction.

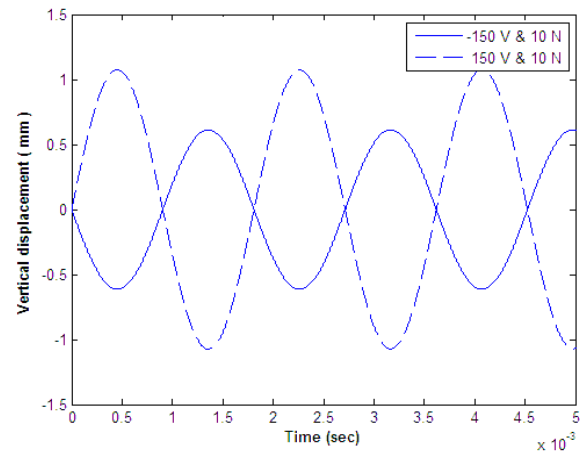

a. Aluminum beam
(AL Substrate -Layer PZT-4)

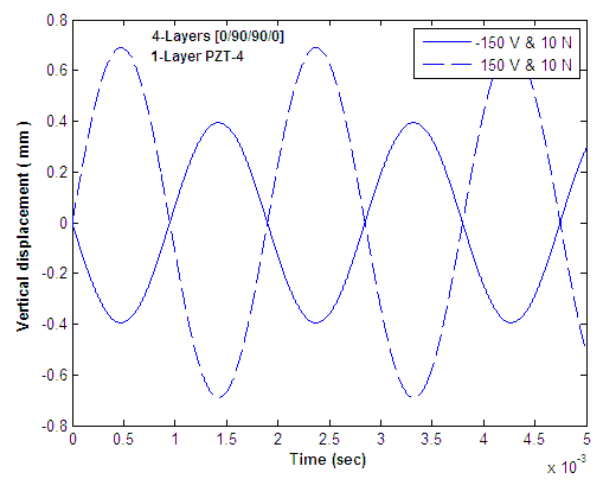

b. Composite beam

Figure 4: Effect of electrical load direction on the beam response.

\section{C: Effect of Electrical Load Amplitude on the Beam Response}

Figure 5 shows the responses of aluminum beam and symmetric angle-ply laminated beam [0/90/90/0] due to the application of electrical loads with various amplitude values $(50,150$, $300 \& 400$ ) volt, and mechanical load with amplitude of $100 \mathrm{~N}$.

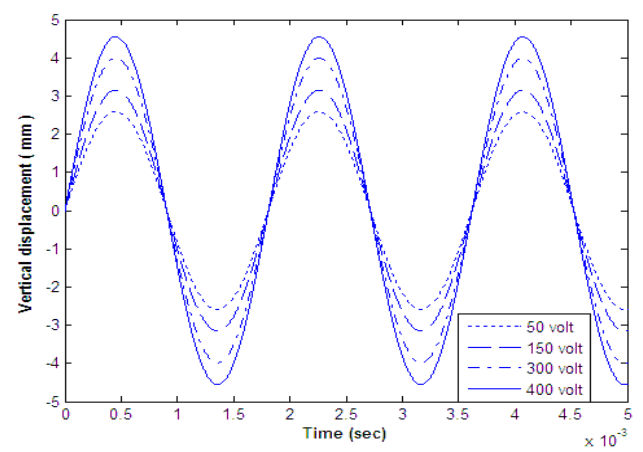

a. Aluminum beam

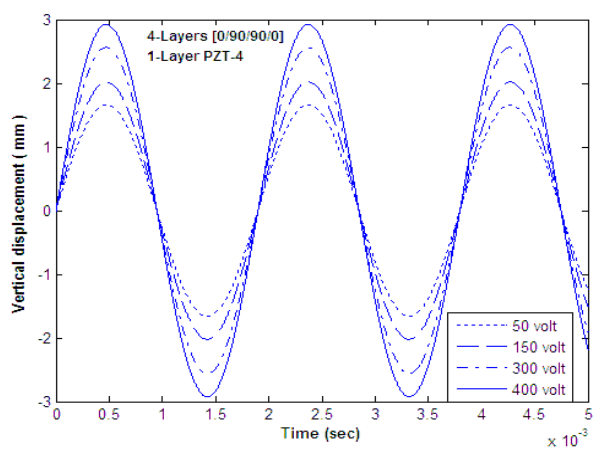

b. Composite beam

(AL Substrate -Layer PZT-4)

Figure 5: Response of beam due to electrical loads

\section{D: Effect of Ply Orientation Angle on the Beam Response}

Figure 6 shows the smart composite beams responses with different ply-orientation angles, symmetric angle-ply laminated beam [0/90/90/0], [30/50/50,30], [40,-45,-45,45], and antisymmetric angle-ply laminated beams [0/90/0/90], [30/50/30,50], [40,-45,45,-45], due to a constant electrical load with amplitude value of 150 volt and mechanical load with amplitude of $100 \mathrm{~N}$. 

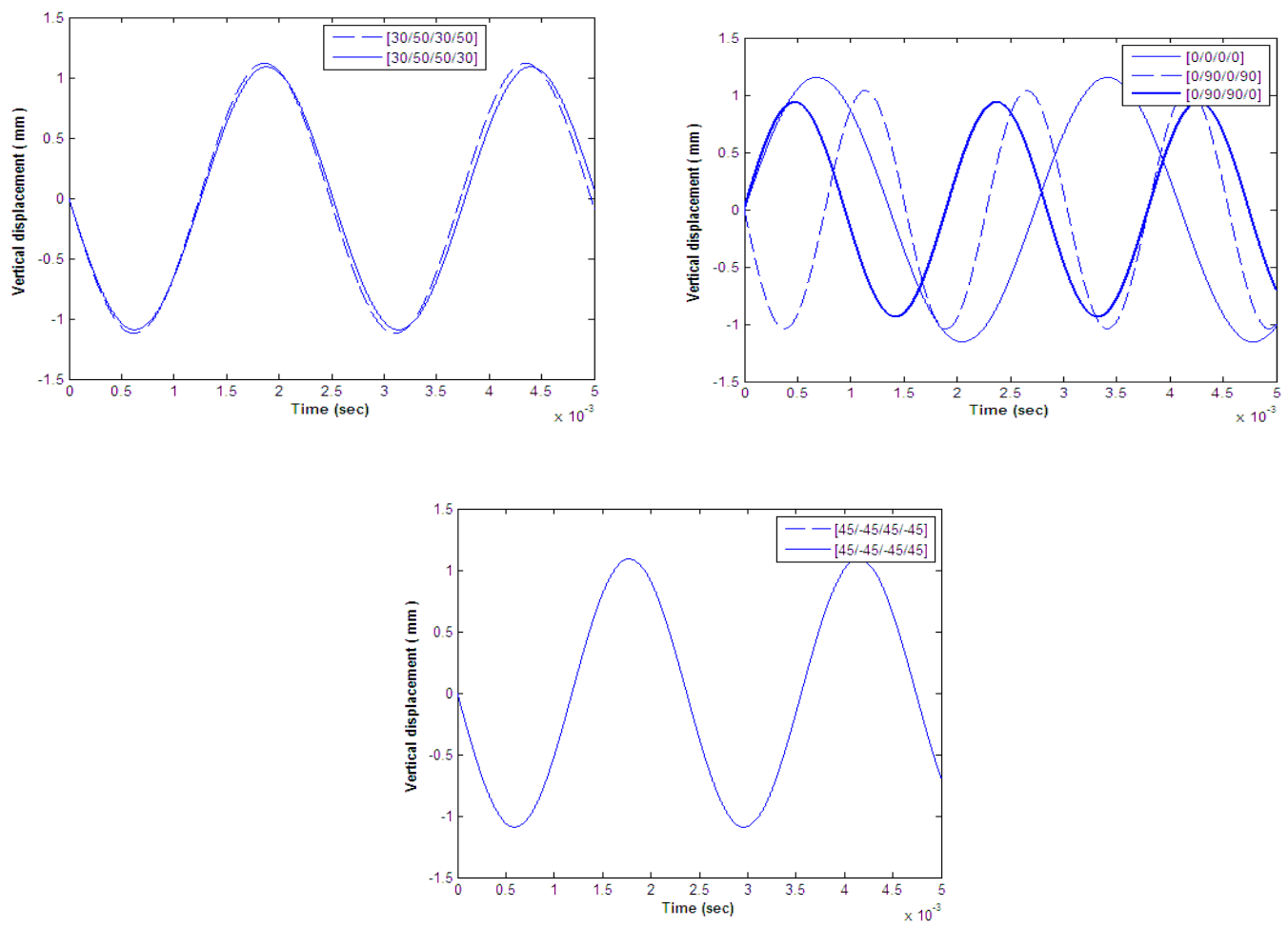

Figure 6: Response of smart composite beam with different ply-orientation angles.

\section{E: Effect of the Loads Application Time on the Beam Response}

Figure 7 shows the effect of the time on the responses of aluminum beam and symmetric angle-ply laminated beam [0/90/90/0] due to the application of electrical load with amplitude of 100 volt and mechanical load with amplitude value equal to $50 \mathrm{~N}$.
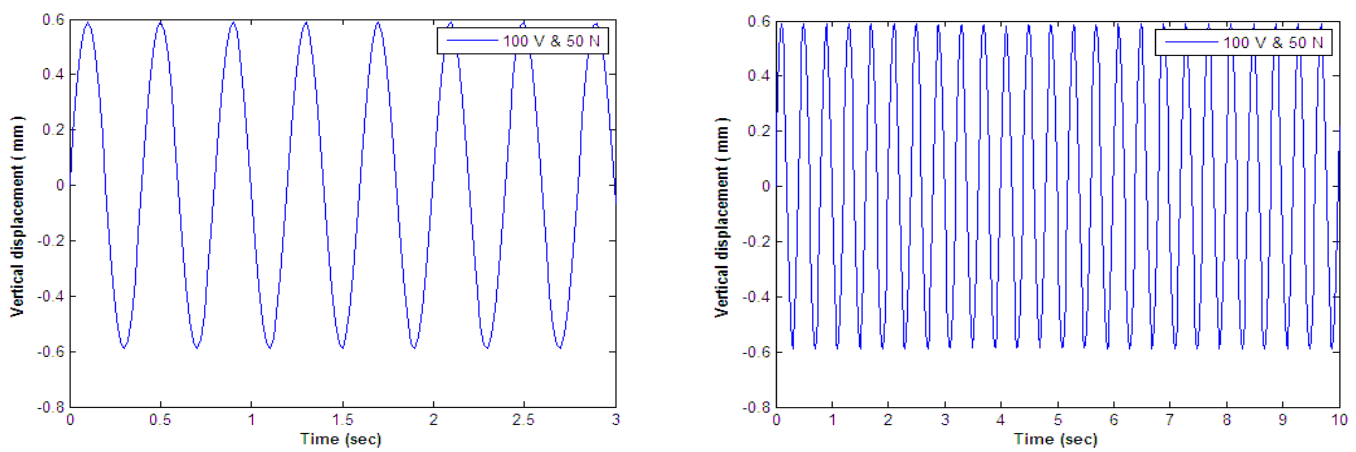

Figure 7: Time response of aluminum beam (substrate 1-layer PZT-4).

\section{F: Beam Response due to the Effect of the Proportional Damping}

The responses of aluminum beam and symmetric angle-ply laminated beam [0/90/90/0] are shown in Figures (8 and 9). Both beams are subjected to electrical load with amplitude 150 volt and mechanical load with amplitude $50 \mathrm{~N}$, and with a time step of $1 \mu \mathrm{s}$ where Rayleigh's coefficients $a=1 \times 10^{-6}, b=0.965 \times 10^{-5}$ (Ramkumar and Ganesan, 2009). 


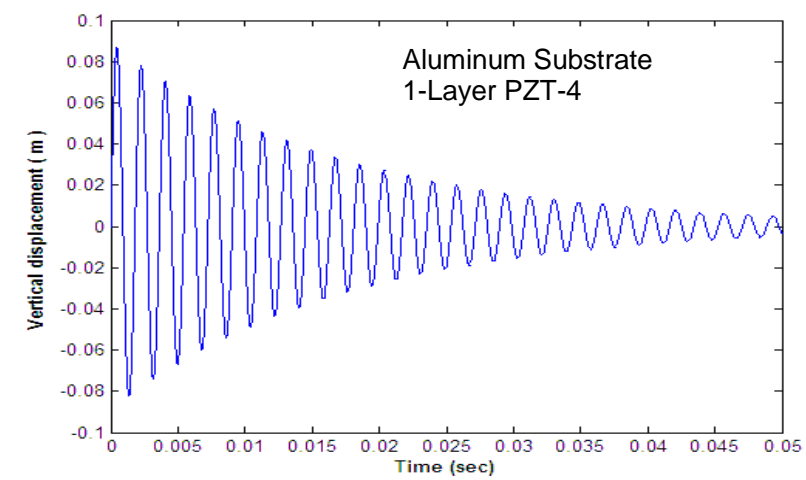

Aluminum beam

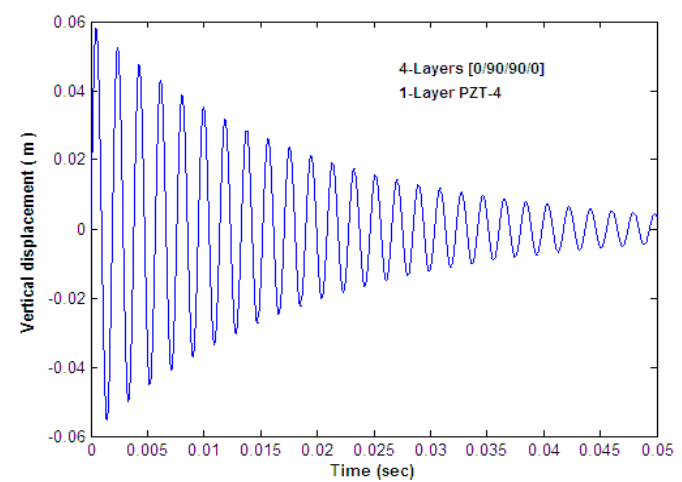

Composite beam

Figure 8: Damped response of aluminum and composite beams

\section{Conclusion}

A finite element model was proposed to predict the static deformation, free vibration characteristics, and the steady state response of laminated aluminum and fiber reinforced composite beams with piezoelectric actuators. The following conclusions have been drawn:

1) The beams responses increase by twice the value as long as the applied mechanical load is applied in the same direction of the electrical loads.

2) The beam responses affected by the direction and amplitude of electric excitation load also the time of application of the electrical loads.

3) Also beams responses affected by the materials of the substrate, ply orientation angles of the fiber reinforced beam, and the chosen value of the damping coefficients.

4) The proposed model did not suffer from the shear correction factor which is problematic in the first order shear deformation theory.

5) A notified decreases of the number of degrees of freedom of the proposed element compared with other element developed by the higher order shear deformation theory, which, of course, save the computational time.

6) The validity of representing the electric potential as a function of the thickness and the length of the beam element in the proposed model.

7) The aluminum beam is responded more than the graphite/epoxy beam as expected because the latter is stiffer in the x-direction. For effective actuation, it is preferable to use piezoelectric with high stiffness in order to import greater actuation capability. In addition the less stiff the substrate is, the greater responding obtained.

\section{References}

[1] Cook R.D., Malkus D. S., and Plesha M.E., 1974, 'Concept and Applications of Finite Element Analysis 3rd Edition', John Wiley \& sons, p. 96, printed in USA.

[2] Elshafei ${ }^{1}$ M. Adnan, Bendary I. M., and Riad A. M., 2010, 'Finite Element Model of Smart Beams with Distributed Piezoelectric Actuators', J. of Intelligent Material Systems and Structures, Vol. 21, pp. 747-758.

[3] Elshafei ${ }^{2}$ M. Adnan and Alraiess Fuzy, 2013. 'Modeling and analysis of smart piezoelectric beams using simple higher order shear deformation Theory', J. of smart material struct, 22(035006(14pp), IOP publisher.

[4] Hwu Chyanbin, Chang W. C., and Gai S. S., 2001, 'Forced Vibration of Composite Sandwich Beams with Piezoelectric Sensors and Actuators' ,4th Pacific International Conference on Aerospace Science and Technology, PICAST4, Kaohsiung, Taiwan. 
[5] IEEE Standard on Piezoelectricity; 1978, 'IEEE Std. 176-1978', The Institute of Electrical and Electronics Engineers, Inc., NY, USA, p.12.

[6] Jones R. M., 1975, 'Mechanics of composite Materials', Hemisphere Publishing Corporation, NY, p40, 51.

[7] Khdeir A. A., and Reddy, J. N., 1997, 'An Exact Solution for the Bending of Thin and Thick Cross-Ply Laminated Beams', Computers and Structures, Vol. 37, pp.195-203.

[8] Khdeir A., and Aldraihem J., 2001, 'Deflection Analysis of Beams With Extension and Shear Piezoelectric Patches Using Discontinuity Functions', Smart Mater. Struct. Vol. 10, pp. 212-220.

[9] Kyu Kang Young, Park Hyun Chul, Hwang Woonbong and Han Kyung Seop, 1996, 'Prediction and Measurement of Modal Damping of Laminated Composite Beams with Piezoceramic Sensor/Actuator', Journal of Intelligent Material Systems and Structures, Vol. 7, pp 25-32.

[10] Mitra Mira, Gopalakrishnan1 S., and Bhat Seetharama M., 2004, ' Vibration Control in a Composite Box Beam with Piezoelectric Actuators', INSTITUTE OF PHYSICS PUBLISHING SMART MATERIALS AND STRUCTURES, Smart Mater. Struct. 13 , 676-690 PII: S0964-1726(04)77133-5.

[11] Nye J. F., 1985,'Physical Properties of Crystals', Oxford Univ. Press. Inc. Printed in the USA, pp.110-115.

[12] Ramkumar. K and Ganesan. N, 2009, 'Finite Element Analysis of Composite Box Structure Containing Piezoelectric Actuators and Sensors for Active Vibration Control', SAE, Noise and Vibration Conf. \& Exhibition, St. Charles, IL, USA, Session: Active Noise and Vibration.

[13] Rao S. S.,1995,'Mechanical vibration-3 ${ }^{\text {rd }}$ Edition', Addison-Wesley Publishing Company, Inc., NY. pp. 425-428; 431-433.

[14] Reddy J. N., 1984, 'A Simple Higher Order Theory for Laminated Composite Plates', Journal of Applied Mechanics, Vol. 51, pp745-752.

[15] Reddy, J.N., 2004, "Mechanics of Laminated Composite Plates and Shells, Theory and Analysis", 2nd Edition, CRC Press, USA, p102.

[16] Samanta B., Ray M. C., Bhattacnaryya R.,1996, 'Finite Element Model for Active Control of Intelligent Structures', AIAA Journal,Vol. 34,No. 9, pp. 1885-1893.

[17] Saravanos D. A., and Heyliger P. R., 1995, 'Coupled Layer wise Analysis of Composite Beams with Embedded Piezoelectric Sensors and Actuators', J. of Intelligent Material Systems and Structures, Vol. 6, pp. 350-363.

[18] Senthil Vel., and Baillargeon P., 2004, 'Active Vibration Suppression of Smart Structures Using Piezoelectric Shear Actuators', 15th. International Conference on Adaptive Structures and Technologies.

[19] Shen M.-H Hereman, 1995, 'A New Modeling Technique for Piezoelectrically Actuated Beams', Computers and Structures, Vol. 75, No.3, pp. 361-366.

[20] Sun Bohua and Huang Da, 2000, 'Analytical Vibration Suppression Analysis of Composite Beams with Piezoelectric Laminae', Smart Mater. Struct. Vol. 9, 2000, pp. 751-760.

[21] Trindade A., and Maio B., 2006, 'Passive Vibration Control of Sandwich Beams Using Shunted Shear Piezoelectric Actuators', CONEM 'IV Congresso Nacional de Engenharia Mecanica'.

[22] Wang Q.A., and Quek S. T., 2000, 'Flexural Vibration Analysis of Sandwich beam Coupled with Piezoelectric Actuator', Smart Materials and Structures 9,103-109.

[23] ZHOU Yan-guo; CHEN Yun-min; and DING Hao-jiang, 2005, 'Analytical Modeling and Free Vibration Analysis of Piezoelectric Bimorphs', Journal of Zhejiang University Science, 6A(9): 938-944. Received Mar. 1, 2005; Revision accepted Apr. 20, 2005. 